\title{
Entrelacs
}

Cinéma et audiovisuel

14 | 2018

Marchés du film : évolutions, mutations et perspectives

\section{Le Festival et le Marché international du film d'animation d'Annecy, un exemple de réconciliation entre la loi du marché et l'intervention institutionnelle}

Jean-Baptiste Scherrer

\section{CpenEdition}

Journals

Édition électronique

URL : http://journals.openedition.org/entrelacs/4290

DOI : 10.4000 /entrelacs.4290

ISSN : 2261-5482

Éditeur

Éditions Téraèdre

Référence électronique

Jean-Baptiste Scherrer, « Le Festival et le Marché international du film d'animation d'Annecy, un exemple de réconciliation entre la loi du marché et l'intervention institutionnelle », Entrelacs [En ligne], 14 | 2018, mis en ligne le 16 décembre 2018, consulté le 22 avril 2019. URL : http://journals.openedition.org/ entrelacs/4290; DOI : 10.4000/entrelacs.4290

Ce document a été généré automatiquement le 22 avril 2019

Tous droits réservés 


\title{
Le Festival et le Marché international du film d'animation d'Annecy, un exemple de réconciliation entre la loi du marché et l'intervention institutionnelle
}

\author{
Jean-Baptiste Scherrer
}

1 Gilles Deleuze, dans l'avant-propos de L'Image-mouvement, déplore la grande vulnérabilité des auteurs de cinéma dont la réalité socio-économique semble concourir à étouffer leur originalité. « L'histoire du cinéma est un long martyrologe ${ }^{1}$ ", écrit-il. Le propos ainsi tenu affirme qu'il ne manque pas de personnes talentueuses et entreprenantes, mais que le poids de l'incertitude du travail créateur conduit à des comportements d'évitement du risque, qu'il soit économique ou artistique, aboutissant à un appauvrissement de la création ${ }^{2}$. Sur le plan théorique et pratique, la France cultive une certaine idée de la diversité et de la politique culturelle. Selon Laurent Creton, la question de savoir s'il est judicieux d'intervenir sur le marché du cinéma ou de le laisser s'autoréguler est « l'un des chantiers les plus importants et les plus exigeants que doivent conduire les sociétés contemporaines ${ }^{3} »$.

2 Les mots « marché » et « institution » entretiennent une proximité conceptuelle qui peut être éclairée par la notion de place. Le marché est associé à l'idée d'une place de marché ; l'institution désigne l'action d'instituer, c'est-à-dire de mettre en place une norme ou une entité de référence. Mais si le marché et l'institution sont tous deux des modes organisationnels, le premier désigne une activité économique spontanée fondée sur la rencontre d'une offre et d'une demande, alors que le second renvoie à une entité ou une norme économique et sociale résultant d'une volonté d'ordre politique.

3 Notre cas d'étude portera sur le Festival et le Marché international du film d'animation d'Annecy. Charles-Clemens Rüling a initié l'étude de l'institutionnalisation du marché (Mifa) et du festival d'Annecy et a appelé à de plus amples recherches pour saisir les 
spécificités du processus institutionnel à l'interface entre l'art et le business ${ }^{4}$. À partir d'une enquête ethnographique ${ }^{5}$, d'interviews et une recherche documentaire, la thèse ici défendue est que le Festival et le Mifa constituent un modèle où la logique du marché et celle de l'interventionnisme institutionnel convergent. En cela, cette manifestation est parvenue à devenir une pièce centrale du monde de l'animation.

Nous proposerons une analyse anthropologique suivant trois perspectives, afin de comprendre la fonction de la manifestation d'Annecy, comme un objet ou un outil fabriqué, comme un ensemble de règles à partir duquel les protagonistes de la manifestation peuvent jouer et enfin comme le réceptacle permettant l'expression d'un désir, d'un rêve.

\section{Le Festival et le Mifa pour les Homo faber}

5 Selon Roger Boussinot dans l'Encyclopédie du cinéma, « un festival peut être un marché aux films, une foire aux vedettes ou une rencontre culturelle, ou les trois à la fois ; chacun d'eux a son visage propre en fonction de son importance, de ses buts, de son idéologie, etc. ${ }^{6}$ » Qu'en est-il ici ? Il est raisonnable d'admettre que cet événement n'existe que parce qu'il parvient à répondre à des attentes particulières.

\section{La position stratégique du Festival et du Mifa, une adaptation au secteur}

6 L'organisation d'événement culturel est l'occasion non seulement de répondre à une stratégie de décentralisation de la politique culturelle, de promotion de l'image et de l'activité économique locale, mais également de fédérer ou d'institutionnaliser une forme culturelle et artistique. La forme organisationnelle du festival s'est imposée. Elle est historiquement dérivée de celle de l'exposition?

7 La ville d'Annecy a accueilli en juin 2018 le 42e Festival et le 28e Marché international du film d'animation. La manifestation a accompagné les évolutions technologiques en termes de production et de diffusion entrainant des transformations, l'apparition du Mifa notamment a été le résultat d'un besoin relatif à la production de films d'animation faisant suite à un plus grand intérêt de la part du public pour le genre animation et à l'apparition de nouvelles chaînes de télévision. En ce qui concerne la production de longs métrages en France, le succès de Kirikou en 1998 a été un tournant en démontrant qu'il était possible de mener des projets rentables sans adapter des bandes dessinées à succès ou se limiter à faire dans l'entertainment ${ }^{8}$. Mais l'élément certainement le plus restructurant est lié aux conséquences de l'arrivée du numérique, notamment sur le mode de fabrication des films. L'animation est devenue « une filière d'excellence pour la France $^{9}$ ». À ce propos, Frédérique Bredin ${ }^{10}$, présidente du Centre national du cinéma et de l'image animée (CNC), a exprimé en 2014 l'idée suivante :

8 Au pays d'Émile Cohl et de Paul Grimault, il est particulièrement réjouissant de voir l'animation devenir aujourd'hui l'un des territoires les plus vivants et les plus innovants du cinéma français : ses succès récents dans les festivals comme à l'exportation témoignent de l'excellence de l'industrie.

9 Dès 1987, en raison de l'influence grandissante du Mifa sur la manifestation, la controverse sur l'évolution identitaire fait rage ${ }^{11}$. La manifestation s'adresse-t-elle à des 
amateurs et des professionnels défenseurs d'une vision auteuriste de l'animation ou à des individus regardant l'animation comme un spectacle grand public? Pour Patrick Eveno et Mickaël Marin, respectivement directeur de la Cité de l'image en mouvement d'Annecy (Citia) et directeur du Mifa, cette manifestation promeut les deux courants. Selon eux, aucune discrimination n'est subie en fonction de l'économie du projet artistique. Ils disent être tout aussi heureux de pouvoir projeter en avant-première une grosse production comme Moi, moche et méchant 3 que de faire découvrir un film comme La Jeune Fille sans mains. Ils défendent l'idée qu'il n'y a pas d'antagonisme entre le Marché et le Festival ou entre l'approche commerciale et artistique. «Peut-être qu'Annecy [ le Festival et le Mifa ] l'a compris un peu plus tôt que les autres. Ce qui fait qu'aujourd'hui, chez nous, c'est complètement naturel et complètement assumé. »

En 2006, Cita été constituée afin d'accueillir le Festival et le Mifa. Son objet est d'être un centre de ressources et de compétences dans le domaine de l'image et des industries créatives, et de fournir des prestations dans les champs de la culture, de l'économie et de la formation. Ceci est cohérent avec la volonté affichée de rechercher des synergies entre le marché et le festival. Les responsables du Mifa, du pôle Rencontres et le délégué artistique, tout en gardant leur indépendance, collaborent étroitement aux dires de Patrick Eveno et Mickaël Marin. La forme juridique de Citia est celle d'un établissement public de coopération culturelle dont l'intérêt majeur est de faciliter l'intervention de l'État et des collectivités territoriales, tout en gardant une autonomie juridique. Ainsi, cette organisation peut en quelque sorte être vu comme une forme d'instance de production. En théorie, un film pourrait être accompagné à tous les niveaux de maturation par le marché et ensuite être sélectionné au festival.

11 La manifestation d'Annecy se positionne comme l'événement le plus important du secteur de l'animation. Les chiffres confirment cette affirmation. Entre 1995 et 2018, la participation la plus basse se fait en 2004 avec 1208 accrédités. Le point le plus haut est en 2018 avec 3800 accrédités soit une augmentation de $214 \%$.

Tableau 1 : Les évolutions chiffrées du Festival et du Mifa

\begin{tabular}{|l|l|}
\hline 2008 & 2018 \\
\hline $\begin{array}{l}\text { Plus de } 500 \text { films projetés et environ 115 } \\
000 \text { tickets vendus }\end{array}$ & Situation sensiblement similaire à celle de 2008 \\
\hline 6700 accrédités & 11700 accrédités (+17\% par rapport à 2017) \\
\hline Approximativement 150 acheteurs & 490 acheteurs \\
\hline 63 pays représentés & 88 pays représentés \\
\hline $\begin{array}{l}219 \text { films choisis par un jury indépendant } \\
\text { pour être en sélection sur } 2200 \text { films } \\
\text { soumis }\end{array}$ & $\begin{array}{l}220 \text { films choisis par un jury indépendant pour } \\
\text { longs métrages (72 en 2017) }\end{array}$ \\
\hline
\end{tabular}

12 Selon le producteur Franck Samuel (La Station Animation), les deux principales rencontres professionnelles du secteur sont celles de Cartoon Movies et du Festival d'Annecy. Or, pour Nicolas Schmerkin, directeur d'Autour de Minuit, la première 
entreprise de distribution de courts métrages d'animation, le Festival de Cannes ou le Festival international du court métrage de Clermont-Ferrand sont tout aussi importants. Tout dépend de l'intérêt porté à la catégorie d'offre (format, genre, cible...). Par ailleurs, des événements similaires à celui d'Annecy ont lieu aux quatre coins du monde. Pour n'en citer que trois : Kidscreen Summit à Miami, le Festival international du film d'animation d'Hiroshima et l'European Animation Awards. Afin de mener une stratégie expansionniste, la marque "Annecy" s'exporte, par exemple avec la création d'un événement en Corée du Sud, et en faisant circuler sa programmation, notamment au Forum des images à Paris, qui a repris le Palmarès d'Annecy les 27 et 28 juin. Par ailleurs, le Festival et le Mifa sont de plus en plus en concurrence avec des événements généralistes. Ceci est sans doute le résultat d'un rapprochement entre le cinéma traditionnel et l'animation. Par exemple, le stand du studio Mikros mettait en avant sa participation à des films de prise de vue réelle comme Au revoir là-haut d'Albert Dupontel. Dernier point, mais pas le moindre, la particularité principale de la manifestation est, selon Mickaël Marin, de combiner le festival et le marché et, de ce fait, de réunir l'ensemble du secteur.

\section{La relativité des intérêts lors du Festival et le Mifa}

13 La participation d'un film à un festival est une bonne chose pour son CV, explique Nicolas Schmerkin. Il aura davantage de chance d'être projeté lors d'autres festivals. Cette circulation est essentielle pour le court métrage, car, contrairement au long métrage et à la série, il s'agit d'un mode de distribution essentiel. De plus, sa notoriété pourra être renforcée par ce qu'en dit la critique et éventuellement en apposant le logo d'Annecy sur l'affiche du film. En outre, les auteurs et les sociétés de production peuvent bénéficier d'aides financières particulières en raison de leur sélection à un festival éligible par le CNC. À titre d'exemple, selon le Code du cinéma, certaines aides à l'écriture de scénario sont conditionnées à la sélection en compétition au sein d'une liste de festivals dont celui d'Annecy fait partie ${ }^{12}$.

Frédérique Bredin déclare que « le Marché international du film d'Annecy s'est imposé en près de trente ans comme un lieu d'échange incontournable où de nombreux projets voient le jour ${ }^{13}$ ». L'objectif du Mifa est de réunir à la fois des personnes désireuses d'obtenir un monopole d'exploitation sur une certaine durée et un certain territoire, des prestataires et des porteurs de projets. En se promenant dans le Mifa, on peut avoir l'impression que tout le monde de l'animation y est représenté. L'événement attire les écoles d'animation, les studios, les représentants de l'animation de différents pays, et tout organisme qui souhaiterait se faire mieux connaître (société de logiciels, représentant de festivals étrangers...). On y trouve aussi des organisations dont l'objectif est de participer à la structuration du secteur, comme des syndicats professionnels, des associations, des clusters, des résidences d'artistes, le CNC...

En quelque sorte, le Festival et le Mifa prennent la forme d'un forum ou d'une agora. Lors des conférences ou simplement en remettant des prospectus, les professionnels du secteur ont l'occasion de partager des points de vue, par exemple sur les contraintes imposées aux scénaristes au nom de la protection de la jeunesse. Lors de l'édition de juin 2018, l'annonce de l'abandon de la fréquence de France 4 a provoqué un grand émoi. Des goodies distribués dans le Mifa communiquaient des slogans de protestations. En outre, les 
questions de la place des femmes dans l'industrie, du harcèlement au travail et de l'image des femmes dans les dessins animés ont été soulevées.

Un autre enjeu important de cet événement concerne la recherche de stages, du premier emploi et les évolutions de carrière. Les candidats peuvent se présenter et prendre rendez-vous, via la plateforme numérique accessible aux accrédités, lors de sessions spécifiques ou de manière continuelle aux stands de studio comme Illumination Mac Guff.

Image 1 : Le stand d'Illumination Mac Guff au Mifa

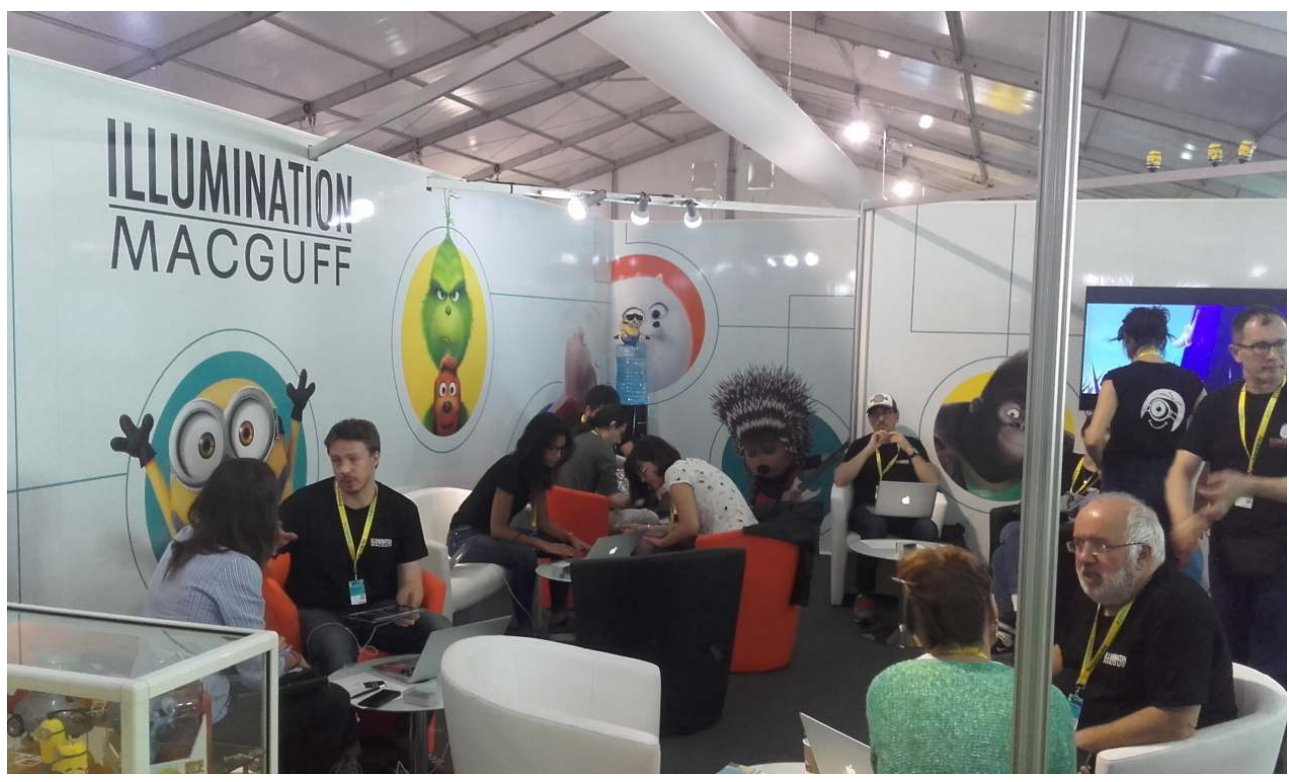

\section{Le Festival et le Mifa pour les Homo ludens}

En 1938, l'historien et théoricien de la culture Johan Huizinga ${ }^{14}$ propose un concept qui connaîtra une fortune considérable : Homo ludens. Il développe l'idée que la dimension sociale du jeu offre un cadre heuristique formidable pour comprendre l'homme. Il est possible d'interpréter l'activité des participants et des organisateurs comme celle d'un jeu particulier dont l'issue est plus ou moins satisfaisante.

\section{Ce à quoi les participants du Festival et du Mifa jouent}

Un premier jeu est de s'approprier un espace pour accueillir ou de se rendre au bon endroit au bon moment. Dans le premier cas, il s'agit généralement de stands qui se situent à l'Impérial, à une quinzaine de minutes à pied du bâtiment principal du festival. Beaucoup d'efforts peuvent être engagés, notamment en termes de décoration: des posters, des vidéos, mais également l'exposition de personnages en trois dimensions. Afin d'économiser des coûts de location de l'espace, la technique de la mutualisation est souvent employée. Ainsi, un producteur polonais pourra donner rendez-vous à un partenaire potentiel au stand de la Pologne.

Que ce soit pour le Festival, le Marché ou les Rencontres, les journées sont scandées par des activités (conférences, projections de films, work in progress, speed dating entre auteurs graphiques et auteurs littéraires...) organisées à l'initiative des participants du Mifa. Le 
but est de cadrer et d'améliorer l'efficacité de certaines tentatives, comme faire connaître des projets à la recherche de financement ou de partenaires, ou encore des éditeurs à la recherche d'un studio intéressé pour adapter leurs œuvres.

Bien entendu, un autre jeu au Mifa est de rechercher des coproductions, vendre ses programmes, chercher des distributeurs à l'international... Mais, contrairement à l'achat d'une peinture lors d'un vernissage, les projets demandent souvent de nombreuses années pour se réaliser. Il faut donc s'apprivoiser les uns les autres, "se renifler» explique Patrick Eveno.

Les festivaliers ne "consomment " pas uniquement des films d'animation, ils espèrent aussi profiter d'une belle expérience. À l'origine, le festival d'Annecy était l'une des rares occasions de visionner des films d'animation. Or, l'animation se diffuse aujourd'hui via les salles obscures, les chaînes de télévision et internet. Selon l'interprétation qu'en donnent Patrick Eveno et Mickaël Marin, l'objectif des festivaliers est de chercher à vivre une expérience singulière en assistant, par exemple, à la projection d'une œuvre inaccessible en salle, d'une avant-première, ou encore via des rencontres avec des artistes, des conférences de presse, des dédicaces... Mais, en raison du nombre limité de places, il faut parfois s'armer de patience, s'organiser, voire renoncer.

Image 2 : Espace du Festival international du film d'animation d'Annecy

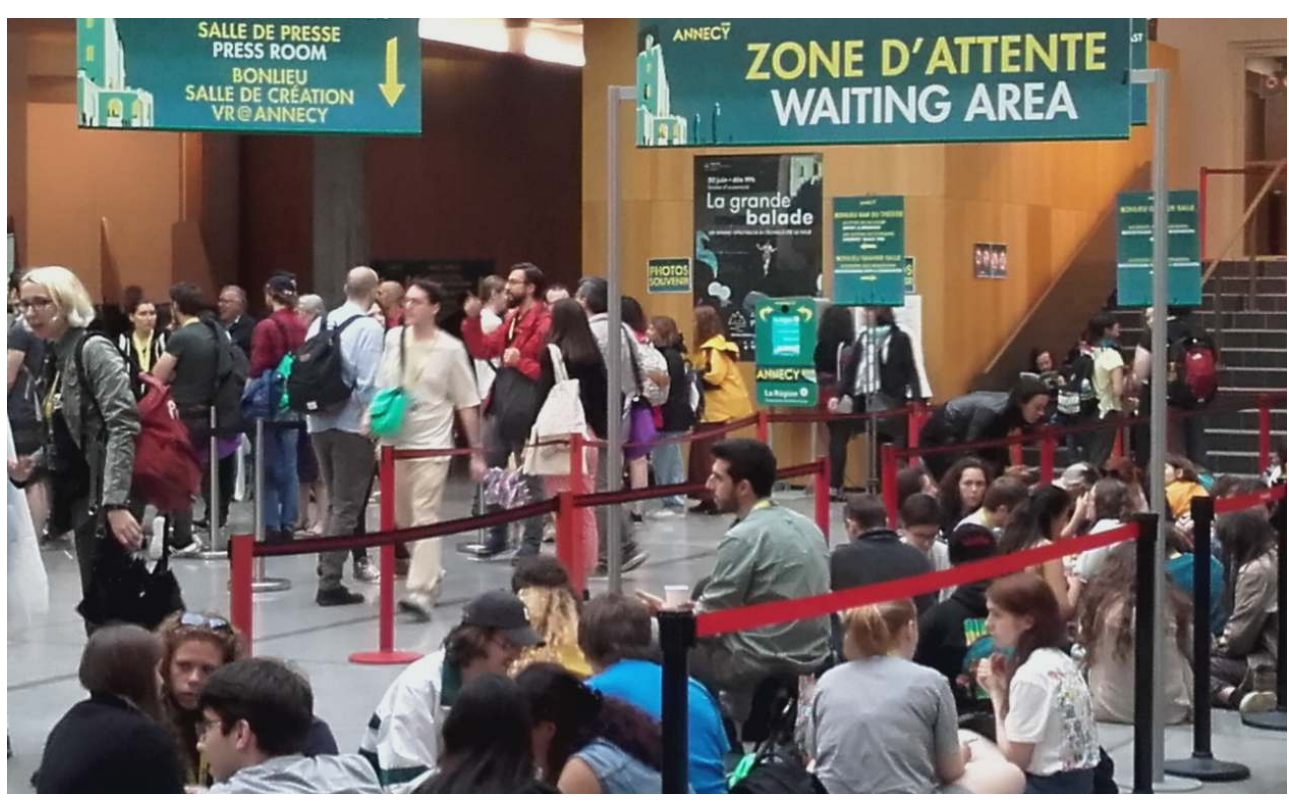

L'enquête ethnographique soutient l'idée que l'événement est vécu de manière très différente selon la catégorie de personnes. Vivre le Festival et le Mifa peut devenir un véritable marathon. Les limites de temps, d'énergie, la richesse de l'offre conduisent à éprouver de la frustration. "Je ne suis pas un robot", confesse un professionnel du secteur, « mais j'aimerais l'être».

\section{Ce à quoi les organisateurs du Festival et du Mifa aspirent}

Faire en sorte que les œuvres, les artistes et les décideurs présents soient nombreux et de qualité est certainement le défi majeur des responsables du Festival et du Mifa. Pour réussir, il faut mener un travail de prospection, participer aux autres rendez-vous de 
l'animation, entrer en contact tout au long de l'année avec de nouveaux interlocuteurs, faire du benchmark ING, rencontrer de nouveaux artistes... Comme dans la majorité de ce genre d'événement, un dispositif est mis en place pour faciliter les prises de contact en amont, et des outils sont mis à la disposition des participants. Par exemple, pour favoriser l'adaptation d'ouvrages littéraires en animation, le Mifa a proposé pour la première fois Shoot the Book Anim', un moment où des auteurs peuvent " pitcher », c'est-à-dire présenter brièvement leurs livres à un public de professionnels de l'animation.

Même s'il est vrai qu'en raison de sa position concurrentielle et par effet de réseau, la manifestation attire mécaniquement de nouveaux participants, la possibilité d'une dégradation de la situation ne peut être écartée. D’une part, sa réussite dépend pour beaucoup de la santé du secteur. D'autre part, l'animation reste encore parfois cataloguée comme un art mineur ou destiné aux enfants. À l'opposé, des chercheurs en esthétique comme Paul Wells y voit plutôt « la forme artistique la plus créative du vingt et unième siècle ${ }^{15}$ ». Malgré l'effet positif que peut apporter le Mifa et le Festival à la réussite commerciale d'un film, notamment pour le lauréat du Cristal du long métrage, la plus haute récompense, cette contribution ne semble pas encore aussi déterminante que celle du Festival de Cannes.

Ensuite, la méthode d'évaluation des films à sélectionner et les conditions pour concourir sont des points controversés. Le Festival a connu au cours des années 1970 des critiques en raison des sélections jugées trop centrées sur les productions nationales. Des avis ont conduit à une perte de confiance dans le comité de sélection ${ }^{16}$. Pour y remédier, chaque année, une nouvelle équipe de sélection est constituée. Le défaut d'une telle pratique, explique le directeur d'Autour de Minuit, est qu'il est alors très aléatoire de concourir, car les sensibilités changent radicalement d'une année sur l'autre. Ensuite, la sélection peut contraindre le producteur ou le distributeur à privilégier un autre festival. Par exemple, si l'exclusivité n'est pas exigée dans un festival, il serait envisageable de faire concourir le film à d'autres occasions.

Par ailleurs, la compétition de films peut être enrayée par des événements extérieurs. Ainsi, le long métrage Have a Nice Day du réalisateur chinois Liu Jain a dû être exclu de la sélection officielle. Il s'est agi d'un acte de censure, rare, mais qui permet d'illustrer le contrôle que des autorités publiques nationales ou étrangères peuvent avoir sur la diversité culturelle.

En théorie, un film pourrait être accompagné à tous les niveaux de maturation par le marché, et ensuite être sélectionné au festival. Un auteur ou un auxiliaire de la création peut porter différents projets. Par exemple, à plusieurs reprises, les responsables de la société Autour de Minuit ont profité des échanges avec les auteurs sélectionnés au Festival pour être associés à leurs nouveaux projets. Cette effervescence est propice à cela. À un niveau plus général, les pays représentés peuvent trouver le marché et le festival complémentaires. Ainsi, l'organisme de promotion du festival estonien a un stand au Mifa, et une rétrospective de l'animation estonienne est programmée dans le cadre du festival. Le festival a également pour fonction de légitimer des œuvres. Par exemple, en 1993, avant qu'il ne devienne mondialement connu, Hayao Miyazaki a reçu le Prix du long métrage des mains de Jean Giraud, également connu sous le pseudonyme de Mœbius ${ }^{17}$. Un des combats de la manifestation est de contribuer à ce que les habitudes et les mentalités évoluent. Pour donner un exemple, afin d'encourager le développement du marché de l'animation ado-adulte se développe, en 2017, la série Lastman et le film Mutafukaz ont été placés sous le feu des projecteurs. 


\section{Le Festival et le Mifa pour les Homo fantasia}

28

\begin{abstract}
notre quête onirique ${ }^{18}$. L'homme est mû par ce désir d'expérimenter au-delà des frontières. Michel Foucault plaide pour une étude scientifique de ces espaces qu'il nomme hétérotopies, «des utopies qui ont un lieu précis et réel ${ }^{19}$ ». Les enfants les connaissent bien, explique Foucault. Il s'agit d'un espace propice à l'imagination et au rêve diurne. Si le rêve se laisse définir comme l'expression d'un désir, alors l'approche deleuzienne du désir mérite d'être ici mobilisée.
\end{abstract}

Pour Edgar Morin, l'imaginaire est l'essence du cinéma ou plutôt le cinéma est la trace de

\section{La manifestation d'Annecy, une hétérotopie}

" On ne vit pas dans un espace neutre et blanc; on ne vit pas, on ne meurt pas, on n'aime pas dans le rectangle d'une feuille de papier ${ }^{20}$. " L'hétérotopie est « liée le plus souvent à des découpages singuliers du temps ${ }^{21}$ ", à la marge du quotidien, un monde dans un monde. Il semble fort à propos de regarder cet événement comme un fait social total, " c'est-à-dire qu'ils mettent en branle dans certains cas la totalité de la société et de ses institutions ${ }^{22}$ ». La ville vit à un autre rythme. Les films du festival sont projetés dans différents lieux, à savoir onze salles de projection et quatre écrans en plein air. En marchant dans les rues, il est impossible de ne pas croiser constamment des personnes portant ostensiblement le pass du festival ou du marché. Les posters du festival sont partout et les commerces accompagnent l'événement.

Image 3 : Offre spéciale d'un commerce dans la ville d'Annecy

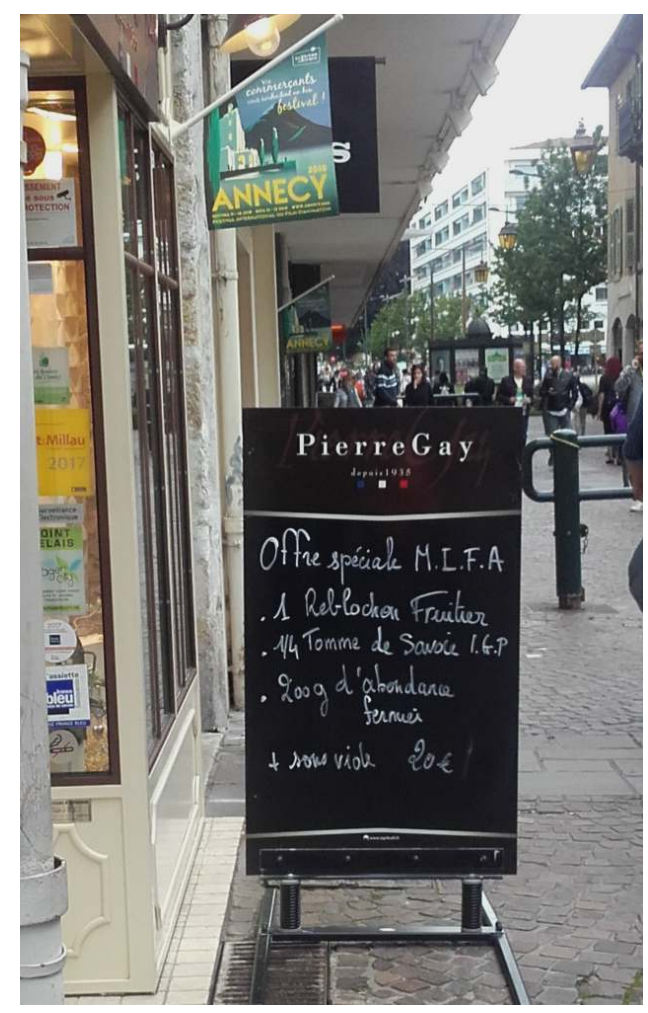


30 Les frontières de la manifestation sont ambiguës. L'essentiel se passe parfois en dehors du cadre tracé. Il y a des espaces cachés ou ésotériques. Pour avoir y avoir accès, on doit souvent être accrédité. Pour cela, il faut remplir certaines conditions, comme être producteur ou journaliste. Un nombre important de bénévoles et un staff de sécurité veillent au respect de ces règles. Selon Foucault, «les hétérotopies ont toujours un système d'ouverture et de fermeture qui les isole par rapport à l'espace environnant ${ }^{23}$ ».

31 Le festival est étymologiquement lié à la fête. Patrick Eveno illustre ce point en décrivant l'atmosphère lors des projections : « Il y a une ambiance de dingue. Des avions en papier volent dans la salle. Et quand le film commence, le silence, très respectueux. » Remarquons que la vidéo Aftermovie de l'édition d'Annecy $2018^{24}$ met entièrement l'accent sur cette dimension festive, voire carnavalesque. Mais il faut préciser que la majorité des fêtes, soirées dansantes, barbecues, petits déjeuners, apéritifs ou autres sont privés. Des organisateurs, comme Netflix ou Disney, sélectionnent par le biais d'invitations les personnes potentiellement intéressantes pour eux. Patrick Eveno nous confie que «ce n'est pas qu'une légende que les meilleurs deals se font autour d'une bière dans une fête improbable ». Mickaël Marin rebondit en soulignant que tout est affaire de confiance. Sans doute, l'effervescence est contagieuse. Désinhibé et mis en situation, la communication de ses rêves, de ses désirs de création qu'on n'ose dévoiler, car précieux et fragile devient alors possible.

\section{Le Festival et le Mifa, une projection au-devant de soi}

La manifestation d'Annecy ne célèbre pas seulement les œuvres passées et présentes, mais prépare les œuvres futures. Une spécificité de la manifestation est l'importance accordée aux étudiants, considérés comme «le sang du festival ». Marcel Jean, délégué artistique du Festival, raconte que, quasiment chaque semaine, il reçoit un message d'une personne expliquant qu'elle était auparavant venue en tant qu'étudiante, que cela avait changé sa vie après y avoir trouvé son premier travail, et qu'elle était aujourd'hui venue proposer un film en compétition ${ }^{25}$. Ainsi, il peut être non pertinent de ranger les participants au sein de catégories figées, car ils sont en devenir. Par exemple, lors d'un sondage auprès de personnes faisant la queue pour assister à une série de présentations de pitchs de film d'animation, l'une d'elles a expliqué que sa motivation était de connaître le principe de ces présentations pour éventuellement en réaliser une l'année suivante.

33 Un aspect difficile à étudier dans cette enquête est l'importance des avortements ou des transformations de projets. Ils sont, par définition, quasiment invisibles. Mais les possibilités offertes par le numérique font qu'il est dorénavant possible de donner accès aux œuvres qui ont concouru, mais qui n'ont pas été sélectionnées. Pour les « chasseurs de trésors » comme Nicolas Schmerkin, il s'agit en quelque sorte d'un Salon des refusés virtuel.

34 À la question de savoir ce qu'est un marché, Michel Callon critique la définition objective et consensuelle offerte par les néo-libéraux et lui substitue une approche dynamique qu'il nomme marché-agencement :

Il n'y a pas de différence entre marché et marketisation. Le fonctionnement des marchés soulève directement et simultanément la question des biens et des identités, qui sont coproduits par des processus de rattachement mutuel. Penser à la marchandisation revient à penser à l'architecture des marchés et à l'organisation de la concurrence, c'est-à-dire aux mécanismes de conception des biens, au formatage des transactions bilatérales et à la singularisation qu'ils impliquent. La 
réflexion politique et morale est au cœur des marchés et n'est pas repoussée à la périphérie ${ }^{26}$.

Le marché n'existe pas a priori, mais il résulte de la confrontation entre agents économiques. Ainsi, la confusion ou la proximité technologique permet au Festival et au Mifa de prendre des parts de marché sur d'autres événements, par exemple ceux autour du jeu vidéo, comme la Gamescom. L'ambiguïté quant à la délimitation de l'animation a amené Marcel Jean, délégué artistique du festival, à créer une catégorie de compétition Off-Limits, c'est-à-dire des films qui interrogent la définition même de ce qu'est l'animation. L'AR (Artificial Reality) et les plateformes en ligne ont maintenant toute leur place. Le festival a vocation à donner un coup de projecteur sur l'ensemble des productions sélectionnées.

Lorsque j'ai commencé à occuper ce poste au festival il y a six ans, ma première impression était que c'était un endroit important pour les grands studios, mais qu'il était très difficile de trouver un endroit pour un travail plus expérimental. C'est la raison pour laquelle j'ai créé la section Off-Limits - ainsi que la section Jeune public et la section Perspective - afin de m'assurer qu'il y avait encore de la place au festival pour ce type de programmation ${ }^{27}$.

\section{Conclusion}

Contrairement à des industries où l'essentiel de la fabrication se situe dans un lieu précis, les activités créatives semblent partout et nulle part. Or, il est indéniable que cet espacetemps particulier constitué lors du Festival et le Mifa est le théâtre d'actions critiques pour la réalisation de projets et de carrière artistique. Interpréter les comportements des auteurs et des auxiliaires de la création comme étant ceux d'un Homo œeconomicus parait peu adapté, d'où notre réflexion sur l'appropriation d'une manifestation comme celle d'Annecy à travers les concepts d'artefact, de jeu et d'imaginaire. Enfin, de plus amples recherches méritent d'être entreprises pour mieux saisir les éléments environnementaux propices à la floraison de films d'animation d'exception. Cette étude se présente comme la première pierre d'un édifice où les arguments de différentes natures, notamment esthétique et économique, deviendraient possibles. Le marché ne devrait pas être le point de départ de ces pourparlers, mais une ligne d'horizon.

\section{BIBLIOGRAPHIE}

Roger Boussinot, L'Encyclopédie du cinéma, Paris, Bordas, 1980.

Michel Callon, « Revisiting Marketization: from Interface-Markets to Market-Agencements ", Consumption Markets \& Culture, vol. 19, n 1, 2016.

Laurent Creton, Économie du cinéma : perspectives stratégiques, Paris, Armand Colin, 2009.

Gilles Deleuze, L'Image-mouvement, Paris, Les Éditions de Minuit, 1983. 
Michel Foucault, Le Corps utopique, Les Hétérotopies, Nouvelles Éditions Lignes, 2012.

Johan Huizinga, Homo ludens : Essai sur la fonction sociale du jeu, Paris, Gallimard, 1988.

Marcel Mauss, Essai sur le don. Forme et raison de l'échange dans les sociétés primitives, La République des lettres, 2013.

Pierre-Michel Menger, Le Travail créateur. S'accomplir dans l'incertain, Paris, Seuil, 2014.

Edgar Morin, Le Cinéma ou l'Homme imaginaire. Essai d'anthropologie sociologique, Paris, Les Éditions de Minuit, 2013.

Cécile Noesser, La Résistible Ascension du cinéma d'animation : socio-genèse d'un cinéma-bis en France (1950-2010), Paris, L'Harmattan, 2016.

Dominique Puthod, Le Festival international du film d'animation : 50 ans d'une histoire animée, Chambéry, Université de Savoie, 2015.

Charle-Clément Rüling, « Event Institutionalization and Maintenance: the Annecy Animation Festival 1960-2010 », dans B. Moeran, J.S. Pedersen (dir.), Negotiating Values in the Creative Industries: Fairs, Festivals and Competitive Events, New York, Cambridge University Press, 2011.

Paul Wells, Animation: Genre and Authorship, London, New York, Wallflower, 2002.

\section{NOTES}

1. Gilles Deleuze, L'Image-mouvement, Paris, Les Éditions de Minuit, 1983, p.8.

2. Selon Pierre-Michel Menger, c'est justement « l'incertitude sur le cours de l'activité et son résultat qui est la condition de l'invention originale, et de 1'innovation à plus longue portée. ", Pierre-Michel Menger, Le Travail créateur. S'accomplir dans l'incertain, Paris, Seuil, 2014, p.11.

3. Laurent Creton, Économie du cinéma : perspectives stratégiques, Paris, Armand Colin, 2009, p.14.

4. Charle-Clément Rüling, «Event Institutionalization and Maintenance: the Annecy Animation Festival 1960-2010 ", dans B. Moeran, J.S. Pedersen (dir.), Negotiating Values in the Creative Industries: Fairs, Festivals and Competitive Events, New York, Cambridge University Press, 2011.

5. À Annecy lors des éditions 2009 et 2018. En particulier, Nicolas Schmerkin (Direction générale, Producteur, Acquisitions) et Annabel Sebag, responsables de la distribution et des ventes internationales, de la société de production et de distribution Autour de Minuit, société dont le cœur de métier est de distribuer des films d'art et d'essai, avec une forte présence au Festival, ont été interviewés fin 2017. Début 2018, des responsables du Festival et du Mifa, Patrick Eveno et Mickaël Marin, nous ont également accordé une interview. Enfin, Franck Samuel, producteur au studio La Station Animation, a apporté sa réflexion et a contribué à cadrer la présente recherche.

6. Roger Boussinot, L'Encyclopédie du cinéma, Paris, Bordas, 1980, p. 481.

7. La Mostra de Venise (Mostra internazionale d'arte cinematografica di Venezia) serait née en 1932 d'une idée du comte Giuseppe Volpi di Misurata, président de la Biennale de Venise, et de la compétition de films au sein de l'Exposition universelle de Paris en 1937. Roger Boussinot, Ibid. p. 230.

8. Voir à ce propos l'ouvrage de Cécile Noesser, La Résistible Ascension du cinéma d'animation : sociogenèse d'un cinéma-bis en France (1950-2010), Paris, L'Harmattan, 2016.

9. https://www.cnc.fr/professionnels/communiques-de-presse/lanimation-une-filieredexcellence-pour-la-france_571984 
10. Extrait des propos de Frédérique Bredin exprimés dans le Guide Mifa 2014.

11. Dominique Puthod, Le Festival international du film d'animation: 50 ans d'une histoire animée, Chambéry, Université de Savoie, 2015, p. 61.

12. Code du cinéma et de l'image animée - Article Annexe 2-14.

13. Extrait des propos de Frédérique Bredin exprimés dans le Guide Mifa 2014.

14. Johan Huizinga, Homo ludens : Essai sur la fonction sociale du jeu, Paris, Gallimard, 1988.

15. Paul Wells, Animation: Genre and Authorship, London, New York, Wallflower, 2002, p. 1.

16. Dominique Puthod, op. cit., p. 37.

17. Dominique Puthod, op. cit., p. 69.

18. Edgar Morin, Le Cinéma ou l'Homme imaginaire. Essai d'anthropologie sociologique, Paris, Les Editions de Minuit, 2013.

19. Michel Foucault, Le Corps utopique, Les Hétérotopies, Nouvelles Éditions Lignes, 2012, p. 23.

20. Michel Foucault, Ibid.

21. Michel Foucault, Ibid.

22. Marcel Mauss, Essai sur le don. Forme et raison de l'échange dans les sociétés primitives, La République des lettres, 2013, p. 102.

23. Michel Foucault, op. cit., p. 32.

24. https://www.youtube.com/watch?v=y8jhhJ4Ugtc\&feature=youtu.be

25. Extrait d'une interview reproduite dans le magazine professionnel Animation World, juin 2018, p. 32 .

26. Michel Callon, «Revisiting Marketization: from Interface-Markets to Market-Agencements ", Consumption Markets \& Culture, vol. 19, $\mathrm{n}^{\circ} 1,2016, \mathrm{p} .18$.

27. Extrait d'une interview reproduite dans le magazine professionnel Animation World, juin 2018, p. 28.

\section{RÉSUMÉS}

Chaque année au mois de juin, la ville d'Annecy accueille des professionnels et des amoureux de l'animation. Le festival et le marché international du film d'animation d'Annecy sont les événements les plus importants du genre. Mais que s'y joue-t-il ? Quelles relations entretiennent le festival et le marché ? Cet article montre comment cet événement participe à l'émergence d'œuvres artistiques et à la vitalité du secteur. Suivant une approche transdisciplinaire, une enquête empirique a été menée pour en saisir le dispositif.

Every year in June, the city of Annecy welcomes professionals and lovers of animation films. The Annecy International Animation Film Festival and Market can be described as the most important event of the genre. But, what is at stake? What is the relationship between the marketplace and the festival? This article shows how the event takes part of the emergence of artworks and vitalise the animation industry. Based on an interdisciplinary approach, an empirical inquiry was undertaken to analyse the apparatus. 


\section{AUTEUR}

\section{JEAN-BAPTISTE SCHERRER}

Titulaire de masters recherche en gestion des organisations (Paris 2), en esthétique du cinéma (Paris 1) et en sociologie (EHESS), il prépare une thèse de droit intitulée Le droit moral et la liberté de création. Étude comparée de droit français, allemand et japonais à l'Université Paris 1 PanthéonSorbonne et l'Université de Fribourg-en-Brisgau. 